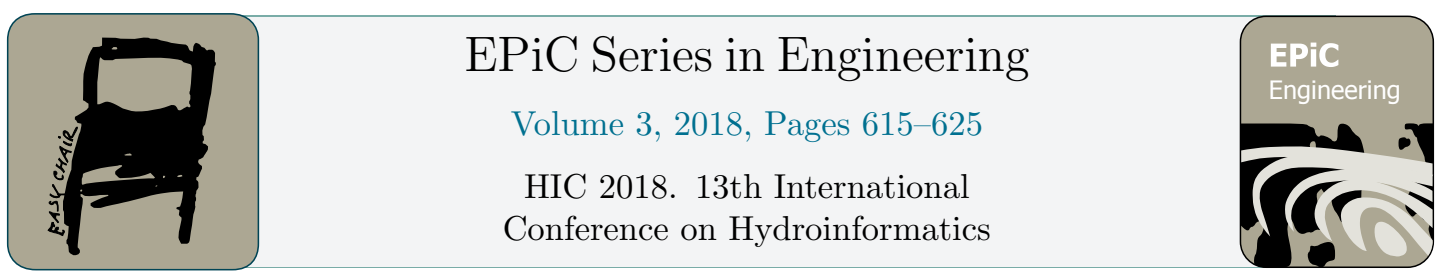

\title{
PIV analysis of cavitating flow behind square multi-orifice plates
}

\author{
Zhiyong Dong, Wenqian Zhao \\ College of Civil Engineering and Architecture at Zhejiang University of Technology, \\ Hangzhou 310023, China \\ Corresponding author: dongzy@zjut.edu.cn
}

\begin{abstract}
Currently, in water supply engineering, the conventional technique of disinfection by chlorination is used to kill pathogenic microorganisms in raw water. However, chlorine reacts with organic compounds in water and generates disinfection byproducts (DBPs) such as trihalomethanes (THMs), haloacetic acids (HAAs) etc. These byproducts are of carcinogenic, teratogenic and mutagenic effects, which seriously threaten human health. Hydrodynamic cavitation is a novel technique of drinking water disinfection without DBPs. Turbulence structures of cavitating flow were observed by the Particle Image Velocimetry (PIV) technique in a self-developed hydrodynamic cavitation device due to square multiorifice plates, including effects of orifice number and orifice layout on velocity distribution, turbulence intensity and Reynolds stress, which aimed at uncovering mechanism of killing pathogenic microorganisms by hydrodynamic cavitation.
\end{abstract}

Keywords: cavitating flow; Reynolds stress; square multi-orifice plate; turbulence intensity; velocity distribution.

\section{Introduction}

With the rapid development of economy and society, drinking water sources have been polluted to different extent. There are various pathogenic microorganisms such as bacteria, virus in raw water, which can infect human being and animals diseases, and be water-borne diseases through effluent of domestic wastewater. In 1894, a bleach technique to kill pathogenic microorganism in raw water was proposed by a German biochemist Traube [1], that is, disinfection by chlorination was used to prevent water-borne diseases, since then it has been being a conventional technique of drinking water disinfection. However, it has been recently found that chlorine reacts with organic compounds in water and generates disinfection byproducts 
(DBPs) such as trihalomethanes (THMs), haloacetic acids (HAAs) etc. These byproducts are of carcinogenic, teratogenic and mutagenic effects [2, 3], which seriously threaten human health. In addition, the cost of chlorination is higher and cycle of disnfection treatment is longer. Therefore, a novel technique of which is both safe and economic has been expected for drinking water disinfection.

Cavitation and cavitation damage phenomena, which contain formation, growth and collapse of cavitation bubbles as well as physical action and chemical reaction of shock wave and microjet, can be enough to disrupt cell of microorganism, break coliform colony and inactivate pathogenic microorganism. Usually, the phenomenon that occurrence of cavitation is based on hydrodynamic principle can be called hydrodynamic cavitation. Disruptions of Baker's yeast and Brewer's yeast cells by hydrodynamic cavitation were experimentally investigated by Save, Pandit and Joshi $[4,5]$, and a comparison with ultrasonic cavitation and mixer were made, only $5-10 \%$ of the energy used by the mixer (blade blender), and the ultrasonic generator horn were used and an efficient large-scale cell disruption of microorganism could be performed. Agaric, actinomycetes and virus killed by hydrodynamic cavitation due to valve-type device were studied by Bodurova and Angelov [6], their results showed that killing rate of hydrodynamic cavitation could reach $71 \% \sim 91 \%$ within $2-4$ min, which was related to cavitation number. The direct effect of cavitation induced by a rotary cavitation device on inactivation of bacteria in water was investigated by Tsenter and Khandarkhayeva [7]. The study showed that hydrodynamic cavitation was a simple and perspective technique and could be potentially used in water disinfection. Experimental study of Escherichia coli killed by hydrodynamic cavitation due to triangular, square and circular multi-orifice plates were carried out by the first author of this paper in the self-developed hydrodynamic cavitation device due to multi-orifice plates, effects of orifice size, orifice number, orifice layout, orifice velocity, cavitation number and cavitation time as well as initial concentration of Escherichia coli on killing rate of Escherichia coli were analyzed [8-10]. This paper aims at experimentally studying turbulence structures of cavitating flow due to square multi-orifice plates so as to uncover mechanism of killing pathogenic microorganisms by hydrodynamic cavitation.

\section{Experimental facility and methodology}

Experimental study was carried out in the Hydraulics Laboratory at Zhejiang University of Technology in China. Experimental setup is shown in Fig.1. The 5 different square multi-orifice plates were designed as shown in Fig.2. The same area of total orifices was remained for each plate. Orifice number $n=9,16,25$ and 64, and the corresponding orifice side length $a=4.5,3.4,2.7$ and $1.7 \mathrm{~mm}$. Checkerboard-type and staggered orifice layouts were designed for 25-orifice plates. Orifice velocity $U=12.49 \sim 27.78 \mathrm{~m} / \mathrm{s}$. Working section of hydrodynamic cavitation due to multi-orifice plates was square cross-section, the height $H=50 \mathrm{~mm}$, and the 
length $L=200 \mathrm{~mm}$. The perspex viewing windows were designed at two sides and top surface in the working section. The Dantec 3D-Particle Image Velocimetry (PIV) System was used in the experimentation. Measuring range in $50 \times 200 \mathrm{~mm}$, acquisition frequency of $15 \mathrm{~Hz}$ and time interval $0.02 \mathrm{~s}$ of instantaneous velocity were taken. Flow field profile flashed by laser light sheet was located at top centreline profile of working section. The $x$ axis was along the longitudinal direction, and the $y$ axis along the vertical direction. The 9 cross-sections were taken along flow direction, $x / L=0.05,0.10,0.15,0.20,0.25,0.35,0.50,0.75$ and 0.90 as shown in Fig.3.

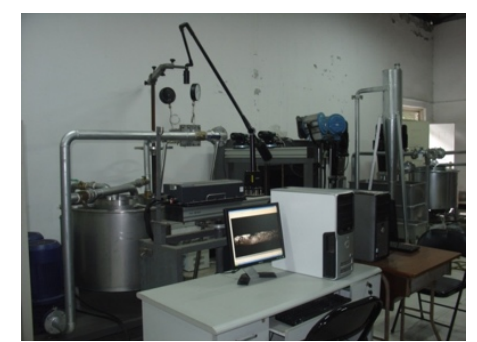

Figure 1 Experimental setup of hydrodynamic cavitation due to multi-orifice plates

64-orifice
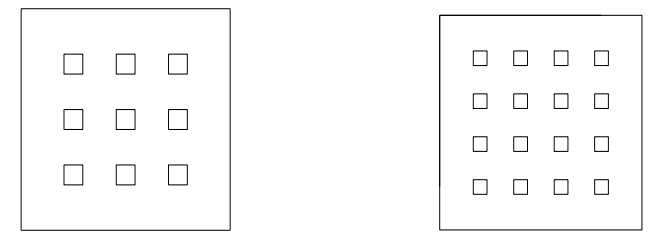

Checkerboard-type 9-orifice Checkerboard-type 16-orifice

\begin{tabular}{|c|c|}
\hline $\begin{array}{lllll}\square & \square & \square & \square & \square \\
\square & \square & \square & \square & \square \\
\square & \square & \square & \square & \square \\
\square & \square & \square & \square & \square \\
\square & \square & \square & \square & \square\end{array}$ & 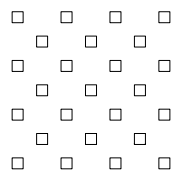 \\
\hline
\end{tabular}

Checkerboard-type 25-orifice Staggered 25-orifice

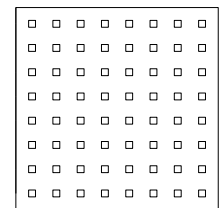

ㅁㅁㅁㅁㅁㅁㅁ

Figure 2 Square multi-orifice plates

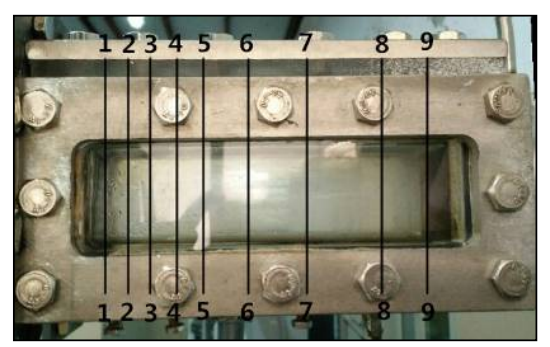

Figure 3 Location of cross-sections behind multi-orifice plate 


\section{Particle image, velocity vector, velocity contour and vorticity}

Taking a 25-orifice checkerboard-type multi-orifice plate for example, orifice velocity $U=14.02$ and $25.61 \mathrm{~m} / \mathrm{s}$, particle image, velocity vector, velocity contour and vorticity of cavitating flow behind the square multi-orifice plate are shown in Fig.4 (a-d). It follows from the Figure that cavitating flow behind the multi-orifice plate is characterized by topological structure, cavitation bubbles are rarer at $U=14.02 \mathrm{~m} / \mathrm{s}$, and denser at $U=25.61 \mathrm{~m} / \mathrm{s}$ and forms cavitation clouds. In fact, high-velocity multiple jets occurred after an incoming flow past a multi-orifice plate, there existed a converging region of multiple jets close to the multi-orifice plate, further downstream, the multiple jets mixed mutually and combined one main flow, and that vorticity accumulated to form vortices due to interaction among cavitation bubbles.

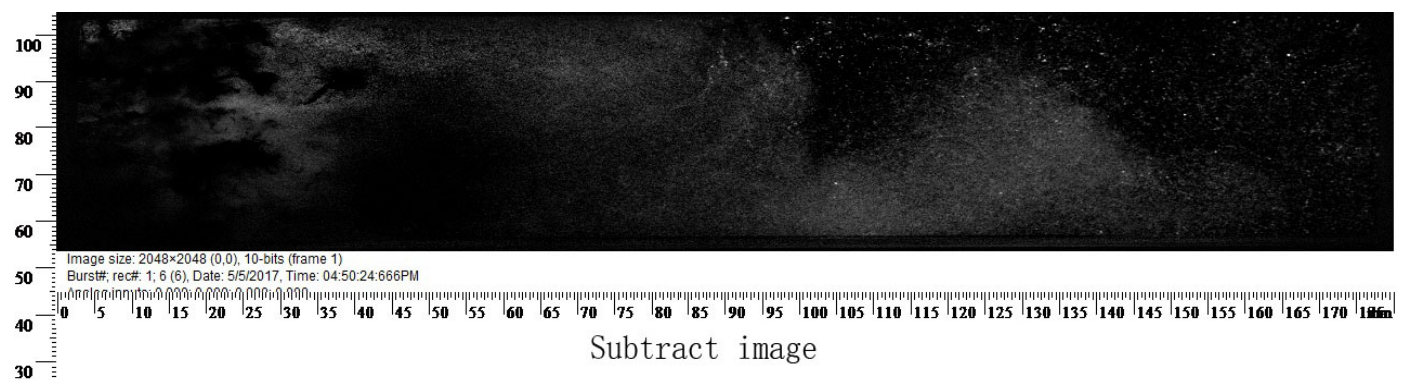

(a) Particle image

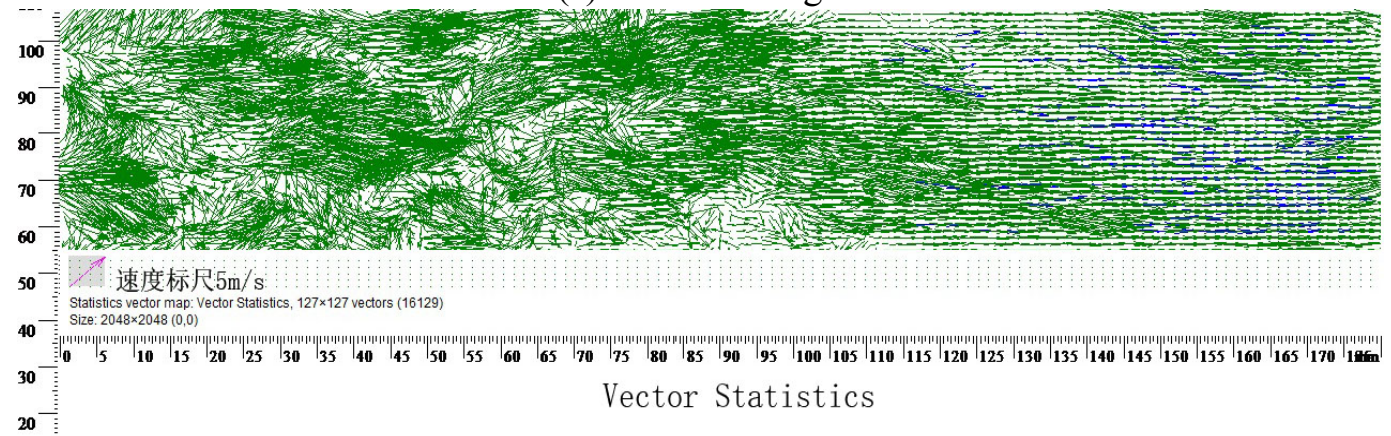

(b) Velocity vector

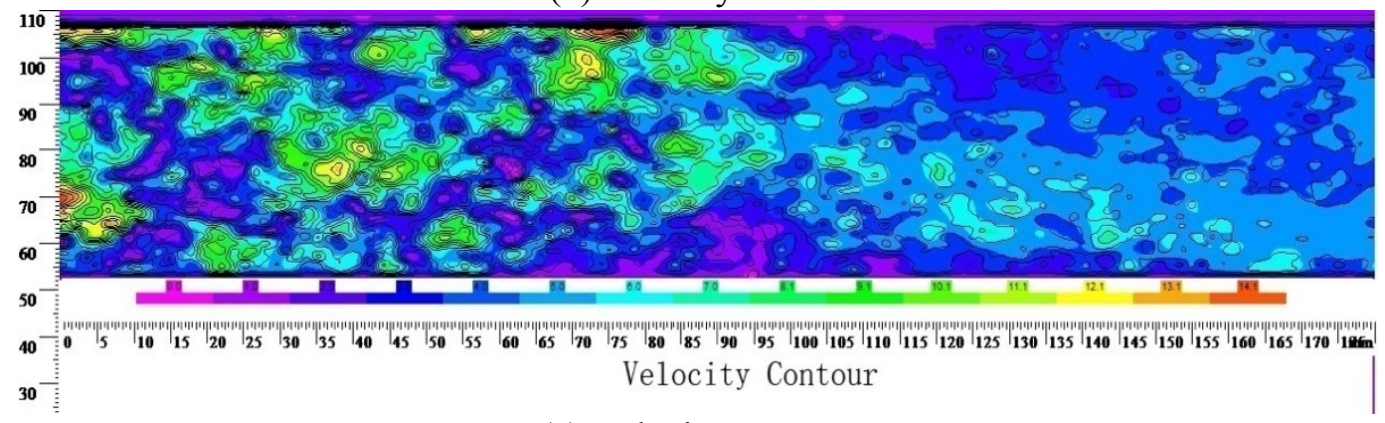

(c) Velocity contour 


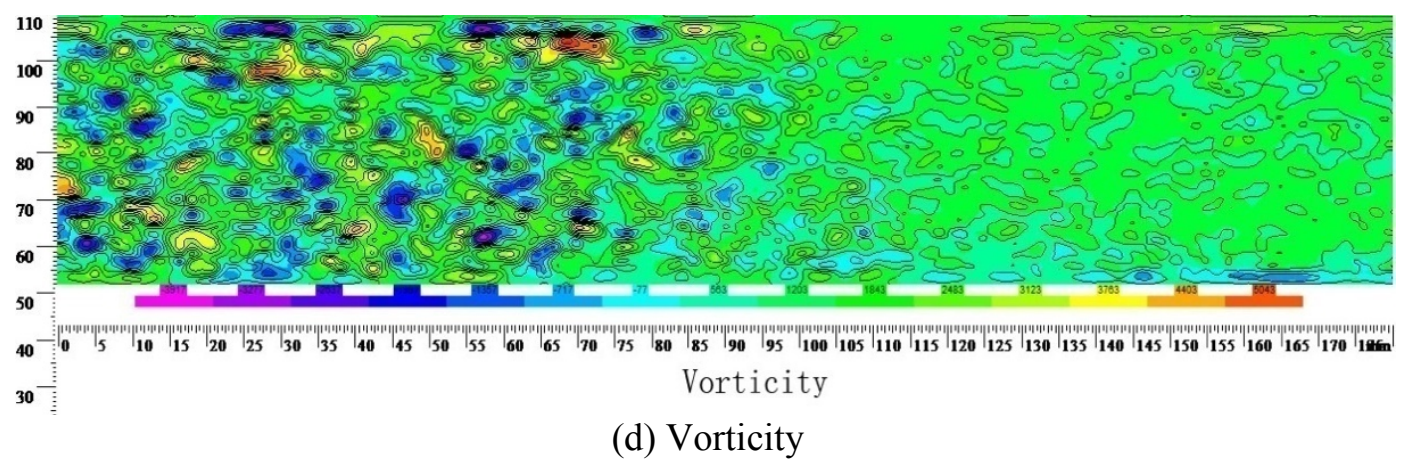

Figure 4 Particle image, velocity vector, velocity contour and vorticity behind a square multi-orifice plate at orifice velocity $U=25.61 \mathrm{~m} / \mathrm{s}$

\section{Longitudinal velocity distribution}

\subsection{Effect of orifice number on velocity distribution}

Longitudinal velocity distribution behind multi-orifice plates is shown in Fig.5. It can be seen that each cross-sectional velocity exhibits sawtooth-like distribution close to multi-orifice plate, see Fig.5(a). Each sawtooth represents one jet because multiple jets occur due to multi-orifice plate. With increase in downstream distance from the plate, the multiple jets merge to flatten the sawtooth-like distribution due to mutual mixing of inter-jet, and combined jets occur. The velocity of combined jets increases with increase in orifice number as shown in Fig.5 (b). Increase in velocity could lower cavitation number and increase number of cavitation bubble, which would intensify shearing effect, thus leading more pathogenic microorganisms to occurrence of cavitation demage and enhancing killing rate [8].

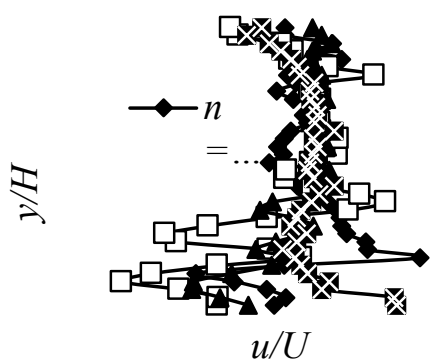

(a) Cross-section $1(x / L=0.05)$

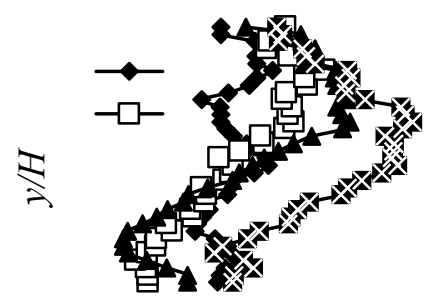

$u / U$

(b) Cross-section $7(x / L=0.5)$

Figure 5 Effect of orifice number on velocity distribution

\subsection{Effect of orifice layout on velocity distribution}

Two multi-orifice plates with the different layouts but the same orifice number are taken for analyzing effect of orifice layout on velocity distribution, which are the 25orifice multi-orifice plates with checkerboard-type and staggered layouts. The velocity with the staggered layout is overall greater than that with the checkerboard- 
type layout close to the multi-orifice plates as shown in Fig.6 (a). On the contrary, with increase in distance from the plate, the velocity with checkerboard-type layout is greater than that with the staggered layout as shown in Fig.6 (b). Multiple jets were formed by the multi-orifice plates, which can be divided into converging region and combined region. The former was close to the plates, the staggered layout enabled multiple jets to generate intense mixing; the latter was far from the plates, the further mixing multiple jets flattened velocity distribution with staggered layout. Shearing effect due to the multiple jets with staggered layout was stronger than that with checkerboard-type layout, which resulted in shearing destroy of pathogenic microorganisms [9].

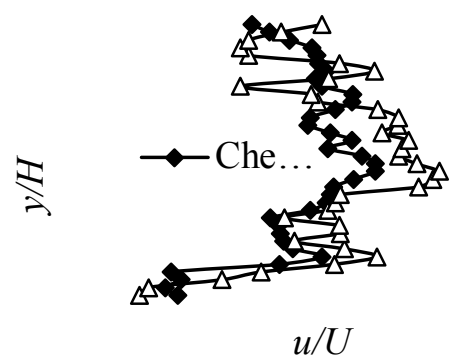

(a) Cross-section $2(x / L=0.1)$

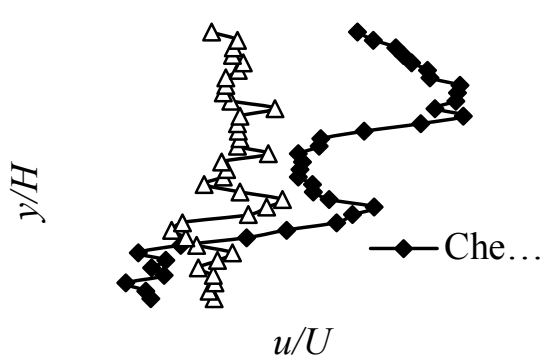

(b) Cross-section $6(x / L=0.35)$

Figure 6 Effect of orifice layout on velocity distribution

\section{Turbulence intensity}

Turbulence level of cavitating flow behind multi-orifice plates can be expressed by turbulence intensity. The longitudinal and vertical turbulence intensities $T_{x}$ and $T_{y}$ corresponding to $x$ and $y$ directions can be respectively defined as

$$
T_{x}=\sqrt{\overline{u^{\prime}}} / U, T_{y}=\sqrt{\overline{v^{\prime 2}}} / U
$$

where $u^{\prime}$ and $v^{\prime}$ denote velocity fluctuations along $x$ and $y$ directions, respectively; $U$ orifice velocity.

\subsection{Comparison between longitudinal and vertical turbulence intensities}

The 9-orifice multi-orifice plate was taken for example, the orifice velocity $U=24.69 \mathrm{~m} / \mathrm{s}$, we can see from Fig. 7 that there are similar magnitudes and patterns between longitudinal and vertical turbulence intensities at the same cross-section. The Poisson equation of pressure fluctuation can be expressed as 


$$
\nabla^{2} \frac{p^{\prime}}{\rho}=-\left[\frac{\partial^{2}\left(V_{i}^{\prime} V_{j}^{\prime}-\overline{V_{i}^{\prime} V_{j}^{\prime}}\right)}{\partial x_{i} \partial x_{j}}+2 \frac{\partial \bar{V}_{i}}{\partial x_{j}} \frac{\partial V_{j}^{\prime}}{\partial x_{i}}\right]
$$

It follows that pressure fluctuation in turbulent flow arises from velocity fluctuation, that is, pressure fluctuation is related to turbulence intensity. It means increase in longitudinal or vertical turbulence intensity enhanced cavitation level of flow, which contributed to kill pathogenic microorganisms.

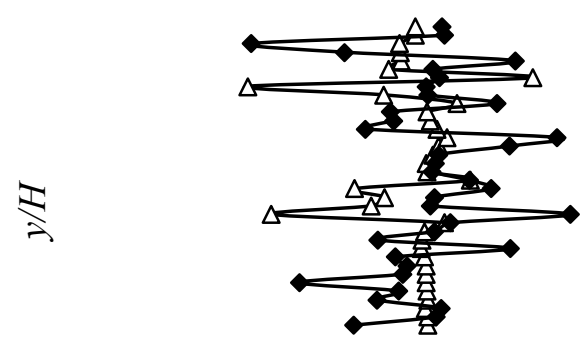

Turbulence intensity

Figure 7 Comparison between longitudinal and vertical turbulence intensities (Crosssection $8, x / L=0.75$ )

\subsection{Effect of orifice number on turbulence intensity}

Effect of orifice number on longitudinal turbulence intensity is shown in Fig.8. It follows from the Figure that the turbulence of cavitating flow both close to the multiorifice plate (see Cross-section 2) and far from the plate (see Cross-section 7) are intense, and further intensified with increase in orifice number. Formation, growth and collapse of cavitation bubble prompt cavitating flow to generate intense turbulence, which means increase in orifice number could contribute to kill more pathogenic microorganisms [8-10].

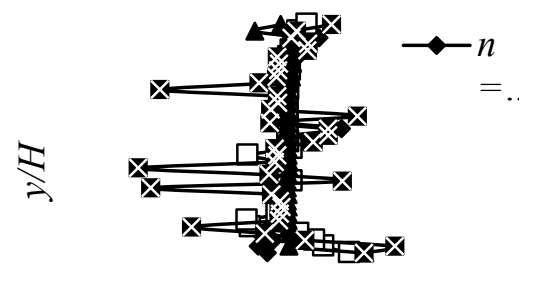

Turbulence intensity

(a) Cross-section $2(x / L=0.1)$

Figure 8 Effect of orifice number on turbulence intensity

\subsection{Effect of orifice layout on turbulence intensity}


Two 25-orifice plates are taken to analyze effect of orifice layout on longitudinal turbulence intensity as shown in Fig.9, which are respectively of checkerboard-type and staggered layouts. It can be found that turbulence intensity of staggered layout close to the multi-orifice plate, i.e., within converging region of multiple jets, is greater than that of checkerboard-type layout, see Cross-section 1. And further downstream, there are similar characteristics of turbulence intensity due to formation of combined region of multiple jets for staggered and checkerboard-type layouts. So killing rate of pathogenic microorganisms was enhanced by the staggered layout of multi-orifice plate [8-10].

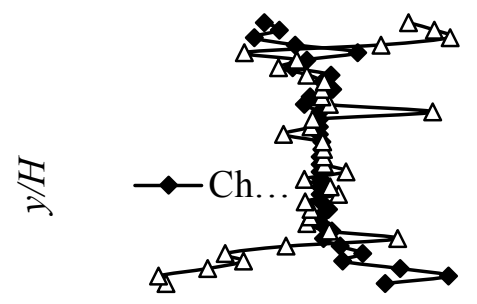

Turbulence intensity

(a) Cross-section $1(x / L=0.05)$

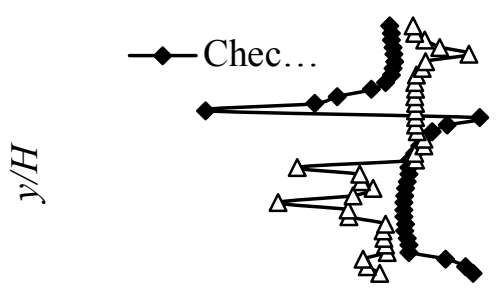

Turbulence intensity

(b) Cross-section $8(x / L=0.75)$

Figure 9 Effect of orifice layout on turbulence intensity

\section{Reynolds stress}

Reynolds stress for longitudinal and vertical velocity fluctuations $u^{\prime}$ and $v^{\prime}$ of cavitating flow behind multi-orifice plates can be expressed as

$$
\tau_{t}=-\rho \overline{u^{\prime} v^{\prime}}
$$

Taking a dimensionless form for the above equation by orifice velocity $U$, we have $\eta=\overline{u^{\prime} v^{\prime}} / U^{2}$

\subsection{Effect of orifice number on Reynolds stress}

As we know, the equation of Reynolds stress can be written as

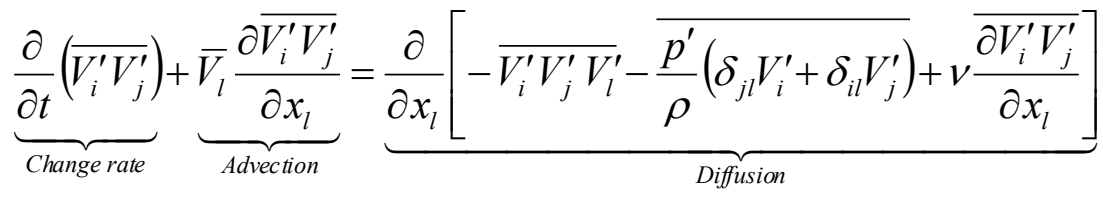

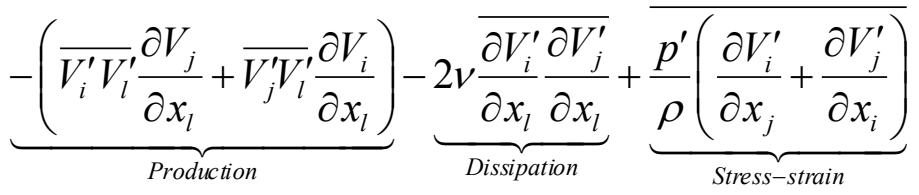


It follows that variation in Reynolds stress depends upon the comprehensive effects due to diffusion, production, dissipation and stress-strain of turbulent flow, which is comparatively complicated. Fig. 10 shows effect of orifice number on Reynolds stress. As can be seen that variation in amplitude of Reynolds stress increases roughly with increase in orifice number. Increase in orifice number could intensify the inter-jet mixing behind multi-orifice plates, thus intensifying the shearing effect due to Reynolds stress, which would contribute to enhance the killing rate of pathogenic microorganisms [8-10].
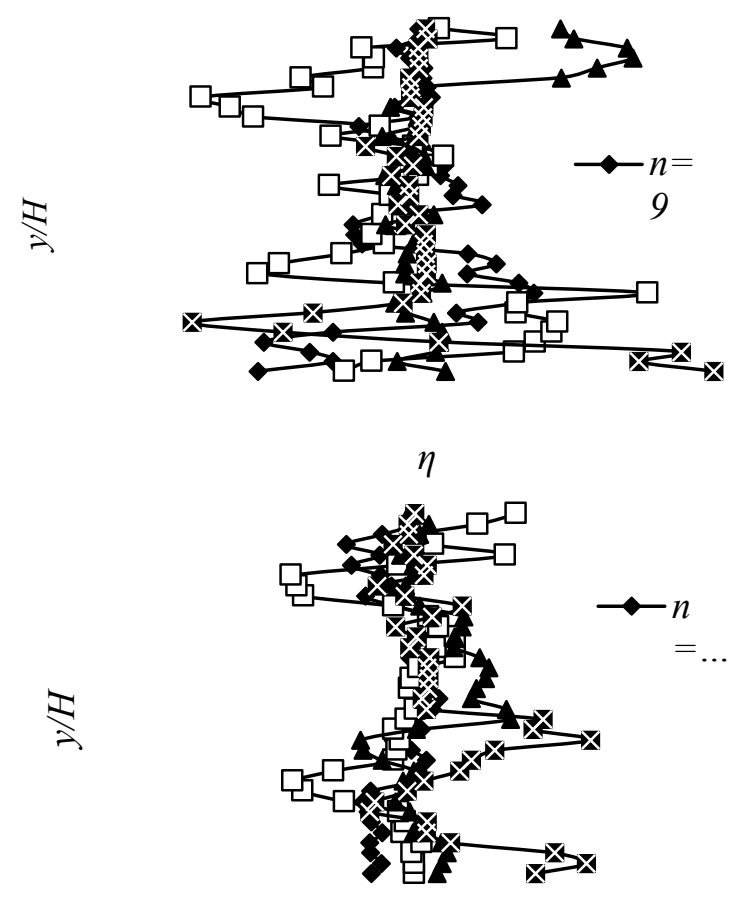

(a) Cross-section $3(x / L=0.15)$

$\eta$ $(x / L=0.5)$

(b) Cross-section 7

Figure 10 Effect of orifice number on Reynolds stress

\subsection{Effect of orifice layout on Reynolds stress}

Similar to the characteristics of turbulence intensity, variation in amplitude of Reynolds stress for the staggered layout close to multi-orifice plate is wider than that for the checkerboard-type layout as shown in Fig.11 (a). And further downstream, there exist similar characteristics for the two layouts as shown in Fig.11 (b). 

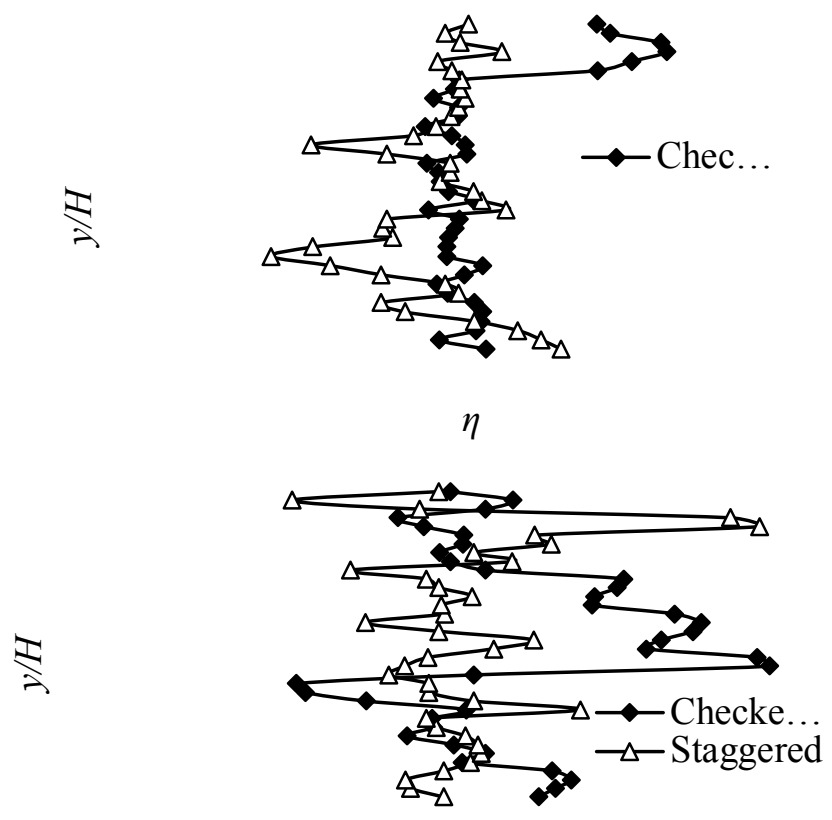

(a)Cross-section $3(x / L=0.15)$

$\eta$

Figure 11 Effect of orifice layout on Reynolds stress

\section{Conclusions}

Through the PIV analyses of cavitating flow fields behind the square multi-orifice plates, we can draw some conclusions as follows:

(1) The high-velocity multiple cavitating jets occurred behind multi-orifice plates, cavitation bubbles were rarer at lower velocity, and dense at higher velocity and formed cavitation clouds. Interaction among cavitation bubbles made vorticity convergence to form vortices. The cavitating flow fields behind multi-orifice plates were characterized by topological structures.

(2) The longitudinal velocity at each cross-section exhibited a sawtooth-like distribution close to the multi-orifice plate, each of which represented one jet. The multiple jets merged to flatten sawtooth-like distribution and formed combined jets. The combined jets gradually increased with increase in orifice number. The velocity for the staggered layout was greater than that for the checkerboard-type layout close to multi-orifice plate. However, with the increase in distance from the plate, the velocity for the checkerboard-type layout was greater than that for the staggered layout.

(3) There were similar magnitudes and patterns for the longitudinal and vertical turbulence intensities at the same cross-section. The turbulence of cavitating flow became intensified with increase in orifice number. The characteristics of turbulence intensity for the staggered layout were similar to that for the checkerboard-type layout far from multi-orifice plate. 
(4) The variation in amplitude of Reynolds stress increased roughly with increase in orifice number. There existed similar characteristics of Reynolds stress for the staggered and checkerboard-type layouts far from multi-orifice plate.

\section{Acknowledgement}

The authors would like to acknowledge the support of the National Natural Science Foundation of China (Grant No.51479177) for this study.

\section{References}

[1] M.Traube, Leben und Wirken des universellen Privatgelehrten und Wegbereiters der physiologischen Chemie, Med. Dissertation, Universitätsbibliothek der Humboldt Universität Berlin, Signatur 94 HB 1449, 1894 (in German).

[2] T. J. Doyle, The association of drinking water source and chlorination by-products with cancer incidence among postmenopausal women in Iowa, American Journal of Public Health. 87 (1997) 1168-1176.

[3] K. L. Simpson, K. P. Hayes, Drinking water disinfection by-products: an Australian perspective, Water Research. 32 (1998) 1522-1528.

[4] S. S. Save, A. B. Pandit, J. B. Joshi, Microbial cell disruption: role of cavitation, The Chemical Engineering Journal. 55(1994) 67-72.

[5] S. S. Save, A. B. Pandit, J. B. Joshi, Use of hydrodynamic cavitation for large scale cell disruption, Chemical Engineering Research and Design. 75 (1997) 41-49.

[6] D. Bodurova, M. Angelov, Intensification the process of water purification by hydrodynamic cavitation, University "Ss Cyril and Methodius"-Skopje Faculty of Mechanical Engineering, Production and Industrial Engineering Association Scientific Conference with International Participation, Manufacturing and Management in 21st Century. Ohrid, the Republic of Macedonia, September, 2004.

[7] I. Tsenter, M. Khandarkhayeva, Effect of hydrodynamic cavitation on microbial inactivation: Potential for disinfection technique, The 13th Meeting of the European Society of Sonochemistry. Lviv, Ukraine, July, 2012.

[8] X. Zhang, Z. Dong, L. Chen et al, Experimental study on Escherichia coli killed by hydrodynamic cavitation behind triangular multi-orifice plates, Journal of Hydroelectric Engineering. 35 (2016) 65-71 (in Chinese).

[9] L. Chen, Z. Dong, C. Liu et al, Experimental study on disinfection of Escherichia coli by hydrodynamic cavitation behind square multi-orifice plates, Journal of Hydroelectric Engineering. 35 (2016) 48-54 (in Chinese).

[10] C. Liu, Z. Dong, L. Chen et al, Experimental study of Escherichia coli killed by hydrodynamic cavitation due to circular multi-orifice plates, China Environmental Science. 36 (2016) 2364-2370 (in Chinese). 